\title{
Wer daran schuld ist und wie man ihr entgeht
}

Wir wagen hier eine düstere Prognose: Am 29. 3. 2010 - also in wenigen Tagen - wird die Zahl der Herzinfarkte um ca. $5 \%$ ansteigen. Das erhöhte Infarktrisiko wird sich auch noch Wochen danach bemerkbar machen. Genau genommen stammt diese Prognose nicht von uns, sondern von Prof. Till Roenneberg vom Zentrum für Chronobiologie der Universität München. Und der hat die Zahlen vom Karolinska Institut in Stockholm. Am 28. 3. werden die Uhren mal wieder vorgestellt. Zahlreiche Studien beweisen, dass die gestohlenen 60 Minuten den Menschen ziemlich belasten.

Der Neurologe Ronald D. Chervin von der University of Michigan schlägt vor, man solle sich langsam an die Sommerzeit herantasten. In der Woche vor der Zeitumstellung könne man jeden Tag 10-15 Minuten früher aufstehen und früher zu Bett gehen. Eine vorweggenommene Zeitumstellung, während der Rest der Welt noch die letzten Tage der Winterzeit genießt? Eine wenig durchdachte Idee.
Wir würden dann am Freitag vor der Zeitumstellung ca. 50 Minuten zu früh aufstehen und müssten abends vor Ende des Spätfilms wieder ins Bett.

Aber es ist nur folgerichtig, dass sich ein Amerikaner Gedanken darüber macht, wie wir die Molesten der Zeitumstellung möglichst folgenlos überstehen. Es waren schließlich auch die Amerikaner, die uns den Unsinn eingebrockt haben. Kein geringerer als der geniale Erfinder und Politiker Benjamin Franklin hat sich nämlich - an einem seiner weniger genialen Tage - die Sache mit der Sommerzeit ausgedacht. Kerzen wollte er sparen, der Knauser. Wir sind nicht die einzigen, die gegen die Sommerzeit opponieren. 75\% der Franzosen sind Sommerzeitgegner. Und unsere Nachbarn verstehen schließlich was von savoir vivre. Nur nützen wird ihnen das nichts. Am Schalthebel der europäischen Uhren sitzen längst die EU-Bürokraten. Die wissen wahrscheinlich auch nicht, wozu die Zeitumstellung gut ist. Aber wer weiß, wie viele Aktenordner selbst der einfachste gesetzgeberische Vorgang im EU-Parlament füllt, wird verstehen,

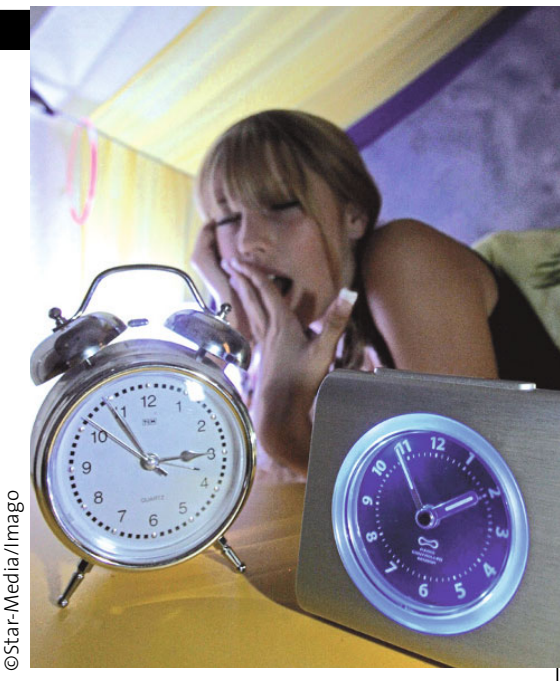

dass die EU die Sommerzeit nicht vor dem nächsten Millennium wieder abschaffen kann.

Einige wenige Völker dieser Erde entziehen sich übrigens konsequent der Sommerzeit. Zu den wenigen Standhaften gehören die Japaner und die Hopiindianer. Nun arbeiten sich Japaner bekanntlich auch ohne Sommerzeit hin und wieder tot. Bleiben also die Hopis. Aber wie wird man Hopiindianer? Um baldigste Nachricht wird dringend gebeten.

REINHOLD MANHART .

\section{ANABOLIKAABUSUS}

\section{Beim Muskelprotz machen die Nieren schlapp}

Athleten, die ihren Muskeln mit Anabolika zu mehr Masse und Kraft verhelfen, setzen damit ihre Nierenfunktion aufs

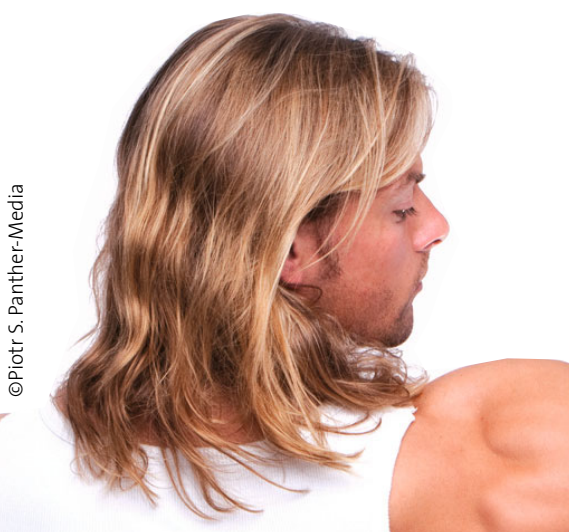

Spiel. Zu diesem Schluss kommt die erste Studie, die den Einfluss eines jahrelangen Anabolikaabusus auf die Nieren bei zehn Bodybuildern untersuchte. Alle hatten über Jahre hinweg ihren Körper mit Steroiden „getrimmt“ und wiesen eine Proteinurie bei stark eingeschränkter Nierenfunktion auf. Bei neun der zehn Kraftath leten wurde eine fokale segmentale Glo- merulosklerose nachgewiesen. Diese „Vernarbung“ tritt charakteristischerweise bei einer chronischen renalen Überlastung auf. Der Befund wies Ähnlichkeiten mit adipositastypischen Nierenschäden auf, war jedoch erheblich stärker ausgeprägt. Die Nieren erholten sich nach dem Absetzen der Steroide.

Eine Ursache für die beobachteten Nierenschäden könnte der extreme Zuwachs an Muskelmasse sein, der die Nieren dazu zwingt, die Filtrationsrate zu erhöhen. Darüber hinaus wird auch ein direkter nephrotoxischer Effekt der Anabolika vermutet.

(Kw) -

42. Jahrestagung der American Society of Nephrology (ASN) 2009 in San Diego/Kalifornien (USA). 